Nervenarzt 2010 $\cdot 81: 523-524$

DOI 10.1007/s00115-009-2852-8

Online publiziert: 3. April 2010

(c) Springer-Verlag 2010

\author{
H.-J. Möller \\ Psychiatrische Klinik der Ludwig-Maximilians-Universität, München
}

\title{
Bipolare affektive Erkrankung
}

heute nicht mehr das klassische, geradezu lehrbuchartige Konzept, dass die bipolare Depression eine Erkrankung ist, bei der akute Krankheitsphasen der einen oder anderen Polarität durch dazwischen liegende symptomfreie Intervalle abgegrenzt sind. Es wird vielmehr darauf abgestellt, dass die Übergänge von dem einen zum anderen syndromatischen Pol sehr fließend sein können und dass diese „mood instability“ der Patienten gerade das Kernphänomen ist.

\section{( "Mood instability“ ist das Kernphänomen der bipolaren Depression} che, dass, obwohl das klinische Erscheinungsbild beider Depressionsformen im Querschnitt sehr ähnlich oder völlig identisch sein kann, doch unterschiedliche medikamentöse Therapieansätze schon bei der Behandlung der akuten Episode berücksichtigt werden müssen.

Im Beitrag zur Epidemiologie, Ätiopathogenese und Verlauf von Bauer et al. wird u. a. deutlich gemacht, dass das gewachsene Verständnis des Krankheitsbildes der bipolaren Depression und insbesondere das damit gewachsene Spektrum der bipolaren Depression dazu geführt hat, dass die heute diskutierten Prävalenzzahlen gegenüber den klassischen Prävalenzzahlen zur manisch-depressiven Erkrankung erheblich angestiegen sind. Statt von einem Prozent wird heute, je nach Enge oder Weite des einbezogenen Spektrums, von einer mehrfach höheren Prävalenzrate ausgegangen. Auch hinsichtlich des Verlaufs dominiert
Dadurch rückten insbesondere die Mischzustände mehr ins Blickfeld und vor allem auch solche Mischzustände, bei denen nur wenig Beimischung vom jeweils anderen Pol vorhanden ist. Es zeigt sich eine deutliche Tendenz, bei bipolaren Erkrankungen von einem wesentlich untraditionell angenommen wurde. Dies hat sicherlich viele Gründe, u. a. auch den der hohen Komorbidität dieser Erkrankung und der Tatsache, dass durch Entwicklungen in der modernen internationalen psychiatrischen Diagnostik sogar stimmungsinkongruente psychotische Symptome als kompatibel mit einer affektiven Erkrankung angesehen werden.

Aus dem Beitrag über das Spektrum klinischer Erscheinungsbilder der bipolaren Depression von Seemüller und Kollegen wird deutlich, wie vielgestaltig die Symptomatik der bipolaren Depression günstigeren Verlauf auszugehen, als dies ist und bei welchen Symptomen u. a. bei einer Erstmanifestation einer Depression bzw. im Querschnittsbild des depressiven Rezidivs, bei der es bisher nicht zu hypomanischen/manischen Episoden kam, eventuell doch von einer bipolaren Erkrankung ausgegangen werden sollte. Insbesondere dem Phänomen des Mischtyps zwischen depressiver und manischer Symptomatik muss dabei besondere Aufmerksamkeit geschenkt werden, da zwar die klassischen Mischbilder, bei denen mehr oder weniger das Vollbild beider Syndrome vorhanden ist, leicht zu diagnostizieren sind, nicht aber Syndrome, bei denen jeweils nur ein oder zwei Symptome vom anderen Syndrompol einbezogen werden. Das Konzept der agitierten Depression, das in Deutschland traditionell als ein Prototyp der endogenen (unipolaren) Depression verstanden wurde, wird zunehmend im Kontext der Diskussion über bipolare Erkrankungen als ggf. dem bipolaren Spektrum zugehörig interpretiert. Die Diskussion hierzu ist noch nicht abgeschlossen, zeigt aber wie schwer es ist, diesbezügliche nosologische Entscheidungen mit ausreichender Eindeutigkeit zu treffen.

Der letzte Beitrag von H. Grunze und S. Dargel beschäftigt sich mit der Akutund Langzeittherapie der bipolaren Depression, wobei aus Platzgründen vorrangig auf die medikamentöse Behandlung eingegangen wird. Dieser Beitrag macht deutlich, dass sich in der Entwicklung der therapeutischen Möglichkeiten und Standards in den letzten 10 Jahren sehr viel getan hat. $\mathrm{Zu}$ erwähnen ist insbesonde- 
re, dass entgegen der früheren Zulassungspraxis inzwischen die Möglichkeit besteht, ein Medikament für die (akute) Behandlung der bipolaren Depression zuzulassen, und von der EMEA entsprechende Leitlinien für die diesbezüglichen Voraussetzungen publiziert wurden. Die erste Substanz, die nach diesen neuen Kriterien eine europäische Zulassung bekommen hat, ist Quetiapin, ein Antipsychotikum aus der Gruppe der neueren Antipsychotika, für das neben der antipsychotischen Wirksamkeit auch eine deutliche antidepressive Wirksamkeit belegt werden konnte. Für eine andere Substanz aus der Gruppe der Stimmungsstabilisierer ist dieser Nachweis nicht in vollem Umfang gelungen, sodass eine Zulassung nicht erreicht werden konnte. Trotzdem gibt es in der metaanalytischen Zusammenfassung der diesbezüglichen plazebokontrollierten randomisierten Studien ein gewisses Wirksamkeitssignal sowie zusätzliche Hinweise aus anderen Studien, die auch dem Lamotrigin einen gewissen Stellenwert in der Therapie der akuten bipolaren Depression beimessen.

Wichtig ist in diesem Kontext die Frage, welchen Stellenwert Antidepressiva in der Behandlung der akuten bipolaren Depression haben. Während in der traditionell deutschsprachigen Psychiatrie ursprünglich ein rein syndromaler Ansatz galt, nachdem bei jeder Form der Depression ein Antidepressivum indiziert ist, hat sich insbesondere in der US-amerikanischen Psychiatrie schon lange eine Tendenz entwickelt, den Antidepressiva bei der Behandlung der akuten bipolaren Depression nur einen, wenn überhaupt, marginalen Stellenwert zuzubilligen und zwar unter zwei Gesichtspunkten:

1. das Switch-Risiko, insbesondere der trizyklischen Antidepressiva, sei zu groß und

2. die Wirksamkeit im Hinblick auf die Behandlung der bipolaren Depression sei nicht ausreichend bewiesen.

Diese Extrempositionen haben in den letzten Jahren immer wieder zu Diskussionen geführt, insbesondere weil als ausreichend effektiv angesehene Alternativen zu den Antidepressiva lange Zeit nicht verfügbar waren. Inzwischen haben sich die Dispute abgeschwächt und wenn man die modernen Therapie-Guidelines anschaut, so findet sich ein sinnvoller Kompromiss. Die bipolare Erkrankung ist eine rezidivierende Erkrankung, deshalb ist im besonderen Maße auch der Langzeittherapie Rechnung zu tragen. Auch hier sind einige neue Entwicklungen zu berücksichtigen.



Hans-Jürgen Möller

\section{Korrespondenzadresse}

Prof. Dr. H.-J. Möller

Psychiatrische Klinik der

Ludwig-Maximilians-Universität

Nussbaumstraße 7,

80336 München

hans-juergen.moeller@med.uni-muenchen.de
VERTIGO XIV Münchner Schwindel-Seminar 2010 Schwerpunkt: Periphere und zentrale Schwindelsyndrome, Okulomotorik- und Gangstörungen

Am 2. und 3. Juli 2010 findet das inzwischen vierzehnte Münchner Vertigo-Seminar unter der wissenschaftlichen Leitung von Prof. M. Strupp, Prof. M. Dieterich, Dr. K. Jahn und Prof. T. Brandt statt. Es wird erstmals gemeinsam von der Neurologischen Klinik und dem Integrierten Forschungsund Behandlungszentrum für Schwindel, Gleichgewichts-und Okulomotorikstörungen (IFBLMU) veranstaltet.

Die Schwerpunkte des Seminars liegen auf der Darstellung klinischer Untersuchungen, insbesondere zur Differenzierung zwischen zentralen und peripheren Schwindelsyndromen, der Rolle der Bildgebung bei der Diagnosestellung sowie dem Verlauf und den Rezidiven bei den verschiedenen Schwindelsyndromen. Aktuelle wissenschaftliche Befunde zur "Ocular tilt reaction" werden ebenso vorgestellt wie Inhalte zum Thema Fahrtauglichkeit von Patienten mit Schwindel. Zudem präsentieren die Veranstalter drei ausgewählte "Schwindel-Fälle" als Videoquiz. Am Ende des ersten Tages ist eine offene Diskussion mit allen Referenten geplant (Fragen hierzu können bereits vorab via EMail an die Veranstalter gesandt werden). Am zweiten Tag werden parallel Kurse mit praktischen Übungen zum Schwerpunkt "Okulomotorische Störungen" angeboten. Diese Kurse sind geeignet für Neurologen, HNO- und Augenärzte, Orthoptistinnen, Physiotherapeuten und MTAs. Sie sollen in die klinischen und apparativen Untersuchungstechniken sowie Therapieverfahren einführen und Kenntnisse sowie praktische Fertigkeiten vertiefen.

Informationen und Ansprechpartner zur Veranstaltung sind unter www.schwindelambulanz-muenchen.de verfügbar.

Veranstalter:

Neurologische Klinik und IFBLMU

Klinikum der Universität München

Campus Großhadern

Marchioninistraße 15, 81377 München

Anmeldeschluss ist der 15. Juni 2010. 